\title{
A 'HAIRY' PROBLEM: TRICHOTILLOMANIA, TRICHOPHAGIA AND TRICHOBEZOARS
}

Singapore Med J 2016; 57(7): 411 doi: 10.11622/smedj.2016125

Dear Sir,

A gastric trichobezoar is an accumulation of hair, usually the patient's own, in the stomach. It can become very large, often presenting with a 'tail' extending into the duodenum; in this form, it is called the Rapunzel syndrome. ${ }^{(1)}$ Gastric trichobezoars most commonly occur in teenage girls with a concomitant, frequently undiagnosed psychiatric disorder known as trichotillomania. Only one-third of these patients have trichophagia and just $1 \%$ ingest enough hair to require surgical intervention. ${ }^{(1)}$ Presentation is usually delayed, often occurring after the ingestion of large amounts of hair over many years.

We herein report a case of a 13-year-old girl who presented with gastric outlet obstruction and symptomatic anaemia. Abdominal examination revealed a vague epigastric mass. On upper endoscopy, a large trichobezoar was identified to be extending beyond the stomach, into the duodenum. Endoscopic removal was attempted but failed. The patient underwent exploratory laparotomy with gastrotomy, which revealed a trichobezoar extending from the greater curvature of the stomach beyond the pylorus, into the duodenum, with a third bezoar concretion $120 \mathrm{~cm}$ from the ileocecal valve. The gastroduodenal and intestinal bezoars were successfully removed via anterior gastrotomy and enterotomy, respectively. A string was seen embedded in the trichobezoar. It snapped during removal of the gastroduodenal bezoars (Fig. 1). Exploration of the peritoneal cavity identified an iatrogenic intussusception of the ileum, which was reduced on enterotomy to retrieve the bezoar in the ileum as well as the remaining string. The patient's postoperative course was uneventful.

Detailed history-taking from her mother revealed that the patient had a past history of pica and trichotillomania. However, her mother had not noticed the compulsive hair-eating behaviour (trichophagia). The patient's identical twin sibling also had pica but was otherwise well. Retrospective examination revealed alopecia

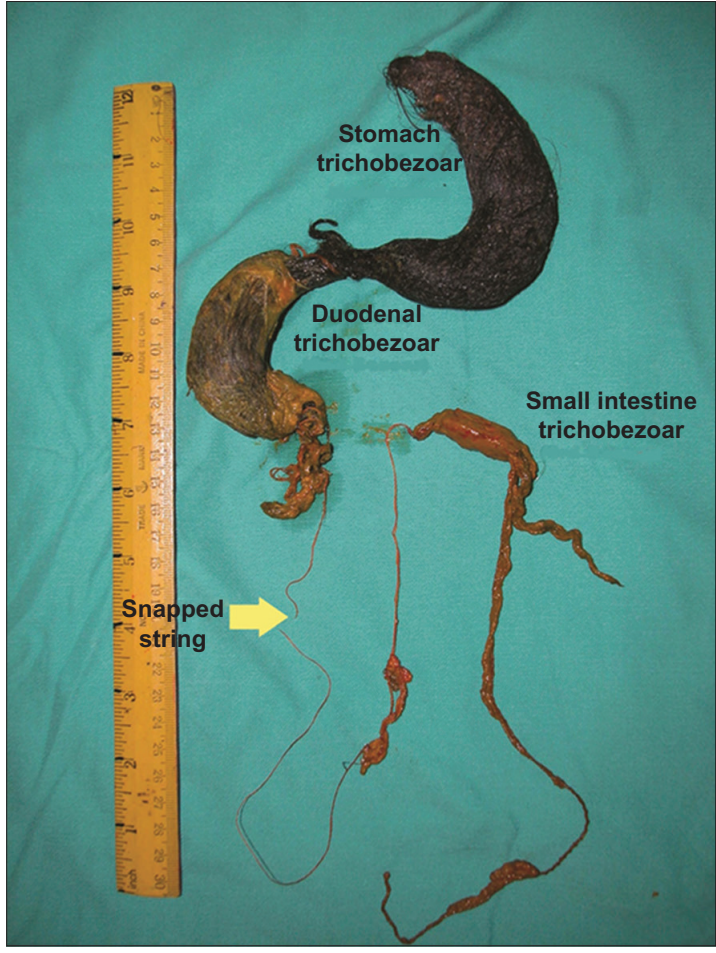

Fig. 1 Photograph shows a large stomach trichobezoar; large duodenal trichobezoar communicating with an embedded, snapped string; and a small intestinal trichobezoar that were completely removed from the patient. areata of the patient's frontal forehead, consistent with trichotillomania. She was referred for psychiatric assessment before discharge. The patient remains well nine years after surgical intervention, with regular psychiatric follow-up.

Radiological investigation, particularly contrast radiography, is a helpful diagnostic means of delineating the anatomical abnormalities created by the trichobezoar, as has been reported. ${ }^{(2)}$ Upper endoscopy has added benefits compared with other investigative modalities. It is very helpful in differentiating a trichobezoar from a phytobezoar, as their individual therapies are different. In contrast to phytobezoars, trichobezoars are resistant to enzymatic or chemical degradation and therefore require removal via endoscopic fragmentation or, for larger trichobezoars, via laparoscopy or laparotomy. ${ }^{(3,4)}$ In this case, laparotomy also allowed for a thorough examination of the peritoneal cavity to assess for distal bezoars along the small and large intestines. Early detection of trichotillomania and trichophagia can prevent this 'hairy' problem; psychiatric treatment is often required and a trichobezoar must be considered as an uncommon differential diagnosis of an epigastric mass in association with trichotillomania and trichophagia.

Yours sincerely,

Yee lan $\underline{\text { Yik }}^{1}$, Ann Kee $\underline{\mathrm{How}}^{2}$

${ }^{1}$ Division of Paediatric and Neonatal Surgery, Department of Surgery, Faculty of Medicine, University of Malaya, Kuala Lumpur, ${ }^{2}$ Department of Medicine, Hospital Raja Permaisuri Bainun, Ipoh, Perak, Malaysia. yiyik@um.edu.my

\section{REFERENCES}

1. Frey AS, McKee M, King RA, Martin A. Hair apparent: Rapunzel syndrome. Am J Psychiatry 2005; 162:242-8.

2. West WM, Duncan ND. CT appearances of the Rapunzel syndrome: an unusual form of bezoar and gastrointestinal obstruction. Pediatr Radiol 1998: 28: 315-6.

3. Gorter RR, Kneepkens CM, Mattens EC, Aronson DC, Heij HA. Management of trichobezoar: case report and literature review. Pediatr Surg Int 2010; 26:457-63.

4. Jatal SN, Jamadar NP, Jadhav B, Siddiqui S, Ingle SB. Extremely unusual case of gastrointestinal trichobezoar. World J Clin Cases 2015; 3:466-9. 